\title{
Sintomatología depresiva en pacientes con fibromialgia
}

\section{Depressive symptoms in fibromyalgia}

\author{
Vanessa Moreno ${ }^{1 a}$, Fernando Namuche ${ }^{1 a}$, Adrián Eduardo Noriega $^{1 a}$, Maritza Vidal $^{2 b}$, \\ Carlos Rueda ${ }^{2 b, 3}$, Jorge Pizarro ${ }^{3 d}$, Luis Vidal2,3c \\ ${ }^{1}$ Facultad de Medicina, Universidad de San Martín de Porres. Lima, Perú. \\ ${ }^{2}$ Centro Diagnóstico de la Osteoporosis y Enfermedades Reumáticas. Lima, Perú. \\ ${ }^{3}$ Hospital Departamental María Auxiliadora. Lima, Perú. \\ a Estudiante de Medicina, ${ }^{\mathrm{b}}$ Médico Asistente, ${ }^{\mathrm{C}}$ Médico Reumatólogo ${ }^{\mathrm{d}}$ Médico Psiquiatra
}

\begin{abstract}
Resumen
Introducción: La fibromialgia es una entidad frecuente en la práctica clínica y se ha considerado que está asociada con desórdenes psiquiátricos, en particular con depresión, lo cual podría tener un impacto en el tratamiento de esta entidad. Objetivo: Evaluar la prevalencia de síntomas depresivos en pacientes con fibromialgia. Diseño: Estudio observacional y de tipo transversal. Lugar: Servicio de Reumatología, Hospital Departamental María Auxiliadora. Participantes: Pacientes de ambos sexos con diagnóstico de fibromialgia. Intervenciones: Se utilizó la escala de Hamilton para la depresión (HDRS) y el recuento de puntos dolorosos para fibromialgia. Principales medidas de resultados: Porcentaje de pacientes con síntomas depresivos. Puntaje total de la HDRS en relación al recuento de puntos dolorosos. Resultados: La edad promedio fue 54,9 \pm 14 años, $80 \%$ fue del sexo femenino y el número promedio de puntos dolorosos, $14,4 \pm$ 1,5. El $32,7 \%$ de pacientes no tenía síntomas de ánimo depresivo, en $27,7 \%$ los síntomas fueron leves, en 30,9\% moderados y solo en $9 \%$ severos. Ningún paciente presentó síntomas compatibles con ánimo depresivo muy severo. Encontramos una correlación significativa entre el número de puntos dolorosos y el puntaje de la escala HDRS ( $r=0,740), p<0,001$. Conclusiones: Los síntomas depresivos severos se encuentran en una proporción baja en pacientes con fibromialgia y parecen estar directamente asociados con el número de puntos dolorosos.

Palabras clave: Fibromialgia; depresión; psiquiatría; dolor.
\end{abstract}

\begin{abstract}
Introduction: Fibromyalgia is a common musculoskeletal problem in clinical practice and is associated with psychiatric disorders. The association with major depression potentially has therapeutic implications. Objective: To determine the prevalence of depressive symptoms in a sample of patient with fibromyalgia. Design: Observational and transversal study. Setting: Rheumatology Department, Maria Auxiliadora Hospital, Lima, Peru. Participants: Fifty-five patients of both sexes with fibromyalgia. Interventions: Fibromyalgia tender points count and use of Hamilton Rating Scale for Depression (HDRS). Main outcome measures: Percentage of depressive symptoms. Total HDRS score in relation to tender point count. Results: Average age was $54,9 \pm 14$ years, $80 \%$ were females, and the average tender point score was $14,4 \pm 1,5$. The HDRS score did not show depression mood symptoms in 32,7\%, 27,7\% had mild symptoms, 30,9\% moderate and 9\% severe symptoms. In no patient depression mood symptoms were considered very severe. There was significant correlation between the number of tender points and HDRS score $(r=0,740), p<0,001$. Conclusions: Severe or very depressive symptoms are infrequent in fibromyalgia and depression mood symptoms seem to correlate positively with the number of tender points.
\end{abstract}

Key word: Fibromyalgia; depression; psychiatry; pain.

\section{INTRODUCCIÓN}

El síndrome de fibromialgia representa una entidad que afecta alrededor de $1 \%$ a 3\% de la población mundial; es una causa común de dolor musculoesquelético crónico en la práctica clínica diaria ${ }^{(1,2)}$. Adicionalmente, se ha reconocido en los últimos años el importante impacto que ejerce la fibromialgia en el paciente, en su familia y en los sistemas de salud; de modo tal que, existe un creciente interés en el estudio de esta entidad ${ }^{(3,4)}$.

La fibromialgia es definida como una forma de reumatismo extraarticular generalizado y que clínicamente se caracteriza por dolor musculoesquelético crónico, asociado con alteraciones del patrón normal del sueño, fatiga y alteraciones psicológicas o de personalidad. El examen físico es negativo, excepto por la presencia de los denominados 'puntos dolorosos', que son áreas anatómicas específicas y predecibles, sensibles a la palpación y de gran utilidad en establecer el diagnóstico ${ }^{(5-7)}$. Al igual que en otras formas de reumatismo extraarticular, las pruebas de laboratorio -incluyendo los reactantes de fase aguda y los exámenes radiológicos-se encuentran dentro de límites normales ${ }^{(6,7)}$.

La etiología del síndrome de fibromialgia aún no está bien definida; pero, la sensibilización central parece tener un papel principal en el desarrollo de esta entidad. Sin embargo, también se ha propuesto factores de naturaleza periférica, alteraciones en neurotransmisores, cambios en aminas vasoactivas y alteraciones psicológicas ${ }^{(8)}$. Al momento actual, se discute ampliamente si en el síndrome de fibromialgia están involucrados mecanismos similares a los del dolor de tipo neuropático ${ }^{(9)}$.
La prevalencia de fibromialgia en la población general y en consultorios médicos no está bien definida, debido probablemente a los diseños de cada estudio en particular. Con respecto a la prevalencia poblacional, en el estudio EPISER en España, diseñado para valorar la prevalencia poblacional de osteoartritis, fibromialgia y artritis reumatoide, se encontró una prevalencia de fibromialgia de 2,4\% en la población general ${ }^{(10)}$. En los Estados Unidos de Norteamérica, se calcula una prevalencia poblacional global de $2 \%$ ( $3,4 \%$ en mujeres y $0,5 \%$ en varones) ${ }^{(1)}$, mientras que estudios provenientes de diferentes países europeos encuentran una prevalencia poblacional que oscila entre $0,7 \%$ y $10,5 \%{ }^{(11)}$.

La prevalencia de fibromialgia se considera elevada en los servicios de atención médica general y especializada. Se calcula 
que en los servicios de atención médica general y familiar la prevalencia de esta entidad se encuentra entre $5 \%$ y $6 \%$ del total de pacientes que acuden a consulta; $y$, en consultorios especializados, la prevalencia oscila entre $10 \%$ y $20 \%$ del total de pacientes ${ }^{(12,13)}$.

En nuestro país aún se desconoce la prevalencia poblacional exacta de fibromialgia, pero existen algunas cifras que conviene comentar. En un estudio poblacional en un barrio urbano-marginal de Lima, se comunicó una prevalencia de $10 \%$ de fibromialgia entre los habitantes encuestados; y, en Piura, se informó una prevalencia de $6 \%$ en pacientes que acudieron a consulta ambulatoria ${ }^{(14,15)}$. Hacen falta estudios poblacionales más extensos que evalúen de manera más exacta en la consulta general y especializada, así como en la población general en nuestro país.

El paciente con fibromialgia característicamente acude a consulta por presentar dolor musculoesquelético crónico, el cual usualmente afecta áreas anatómicas amplias, especialmente en columna cervical, región lumbar y porciones proximales de cintura pélvica y escapular. La naturaleza del dolor hace que el paciente lo describa en términos simples como 'me duele todo el cuerpo' o 'todo el tiempo tengo dolores en diferentes partes del cuerpo'. La mayoría de pacientes refiere además rigidez matutina en manos, que puede durar desde pocos minutos hasta algunas horas ${ }^{(16-18)}$.

En el examen físico en la forma primaria de fibromialgia, a excepción de los 'puntos dolorosos', el resto del examen es normal. Es importante mencionar que, a pesar de los síntomas referidos por el paciente, no se encuentra sinovitis, derrame articular ni signos de daño o lesión articular, a menos que fibromialgia coexista con alguna otra patología que afecta al aparato musculoesquelético ${ }^{(1,16,17,19)}$.

Las alteraciones del patrón normal del sueño son frecuentes en fibromialgia y los pacientes generalmente lo describen como insomnio inicial o terminal, sueño no reparador, intranquilidad nocturna o sueño interrumpido, los mismos que se asocian con fatiga y cansancio matinal. Estos síntomas, conjuntamente con la naturaleza crónica de fibromialgia, afectan la vida personal y laboral de las personas afectadas; y está claramente reconocido que esta entidad representa una causa importante de discapacidad y ausencia laboral a nivel mundial ${ }^{(20-22)}$.

El diagnóstico de fibromialgia se establece sobre bases clínicas y no ofrece mayor dificultad si se toma en cuenta los criterios diagnósticos planteados por el American Rheumatology College, según los cuales se exige que el paciente presente dolor musculoesquelético generalizado con al menos 11 de 18 probables 'puntos dolorosos' ${ }^{(23)}$.

Diferentes estudios han demostrado que los síntomas psicológicos, y en particular la ansiedad y depresión, son comunes en fibromialgia; incluso, en algunos de estos pacientes representa un problema mayor ${ }^{(24,25)}$. Desde que la presencia de depresión como una comorbilidad importante en fibromialgia podría tener implicaciones terapéuticas, el objetivo el presente estudio fue evaluar la presencia y severidad de síntomas depresivos en una población de sujetos con fibromialgia. Asimismo, se ha comunicado que el número total de 'puntos dolorosos' se correlaciona de manera lineal con los síntomas y alteraciones psicológicas en fibromialgia ${ }^{(7)}$, motivo por el cual, el segundo objetivo del presente estudio fue evaluar si existe una correlación positiva entre el recuento de 'puntos dolorosos' y los síntomas de ánimo depresivo en una muestra de pacientes con fibromialgia.

\section{MÉTODOS}

El presente trabajo fue de tipo observacional y de diseño transversal. Se realizó entre los meses de febrero a octubre del año 2009, en el Servicio de Reumatología del Hospital de Apoyo María Auxiliadora, Lima, Perú.

Todos los pacientes que ingresaron a este estudio cumplían los criterios diagnósticos para fibromialgia vigentes del ACR ${ }^{(26)}$, los cuales son:

I. Historia de dolor generalizado. El dolor es considerado como criterio cuando todo lo siguiente está presente: dolor en el lado izquierdo del cuerpo, dolor en el lado derecho del cuerpo, dolor por encima de la cintura, dolor por debajo de la cintura. Adicionalmente, también debe de estar presente dolor en el esqueleto axial (columna cervical, dolor torácico anterior o dolor en la columna torácica o dolor lumbar). En esta definición se considera al dolor en hombros o en los glúteos como dolor en el lado afectado. El dolor lumbar bajo es considerado dolor en el segmento inferior.

II. Dolor en al menos 11 de los 18 puntos dolorosos a la palpación digital. Según esta definición, debe de estar presente dolor a la palpación en al menos 11 de los siguientes 18 puntos dolorosos, considerando que todos los puntos dolorosos son bilaterales:

1. Occipitales: En la inserción de los músculos suboccipitales.

2. Cervicales bajos: En el aspecto anterior del espacio intertransverso a nivel de C5-C7.

3. Trapecio: En el punto medio del borde superior.

4. Supraespinoso: Sobre la espina escapular, cerca del borde medial.

5. Segunda costilla: En la segunda unión condrocostal, justo lateralmente a las uniones de las superficies superiores.

6. Epicóndilo lateral: Dos centímetros distales a los epicóndilos laterales.

7. Glúteos: En el cuadrante superior y externo del glúteo, en la parte anterior del músculo.

8. Trocánter mayor: Posterior a la prominencia trocantérica.

9. Rodillas: En la porción de la almohadilla grasa medial proximal a la línea articular.

Según el Colegio Americano de Reumatología, la palpación digital debe de efectuarse con una fuerza aproximada de $4 \mathrm{~kg}$; para que un punto sea considerado positivo, el sujeto debe de referir que la palpación fue dolorosa. La 'sensibilidad' no es considerada 'dolor'.

Adicionalmente, a los pacientes que ingresaron al estudio se les hizo el recuento de 'puntos dolorosos' en total y se consignó el número que se encontró 
en cada paciente. Una vez establecido el diagnóstico de fibromialgia, tres evaluadores realizaron una entrevista clínica utilizando la escala de Hamilton para la depresión (HDRS). La HDRS es un instrumento de tipo escala y calificada por el observador. Su contenido se centra particularmente en los aspectos somáticos y de comportamiento de la depresión; también valora los síntomas vegetativos, cognitivos y de ansiedad. Esta escala fue diseñada para ser utilizada en pacientes diagnosticados previamente de depresión, con el objetivo de evaluar cuantitativamente la gravedad de los síntomas y valorar los cambios del paciente deprimido ${ }^{(27,28)}$.

La versión original de la HDRS constaba de 21 ítems. Posteriormente, se diseñó una versión reducida con 17 ítems, que es la recomendada por el Instituto Nacional de Salud Mental de los Estados Unidos ${ }^{(27,29)}$. La validación de la versión en castellano de esta escala se realizó en 1986 por Ramos-Brieva ${ }^{(30)}$. Se considera que la HDRS posee una buena consistencia interna (alfa de Cronbach entre 0,76 y 0,92 , según diferentes estudios). La variabilidad interobservador oscila entre 0,65 y 0,9. La validez de la HDRS en relación con otros instrumentos que valoran globalmente la depresión oscila entre 0,8 y 0,9 ; esta escala tiene una sensibilidad de $85 \%$ para detección de depresión en población general y en el área clínica ${ }^{(31)}$.

La puntuación total en la HDRS va desde 0 a 52 y, según la Asociación Americana de Psiquiatría, la categorización de la intensidad o gravedad del trastorno depresivo se realiza mediante la siguiente escala: muy severo $>23$ puntos, severo 19 a 22 puntos, moderado 14 a 18 puntos, leve 8 a 13 puntos y normal 0 a 7 puntos. En el presente estudio empleamos esta escala de calificación para la evaluación del ánimo depresivo en los pacientes con fibromialgia ${ }^{(32)}$.

Para el análisis y tabulación de los datos se utilizó los programas Microsoft Excel y SPSS 16,0 para Windows, empleándose estadística descriptiva para la presentación de datos. Para el análisis estadístico se utilizó la prueba $\mathrm{X}^{2}$, que permite determinar si dos variables cualitativas están o no asociadas. Si al final del estudio concluimos que las variables no están relacionadas, podremos decir con un determinado nivel de confianza, previamente fijado, que ambas son independientes. Asimismo, se utilizó coeficiente de correlación de Pearson para la asociación de puntos dolorosos presentes en cada paciente y el puntaje encontrado en la escala de Hamilton. El nivel de intervalo de confianza que se utilizó fue de $95 \%$.

\section{RESULTADOS}

Evaluamos un total de 55 pacientes con diagnóstico reciente de fibromialgia y que no habían recibido tratamiento previo para esta entidad. En relación al género, 44 pacientes fueron de sexo femenino (80\%) y 11 de sexo masculino (20\%); la edad media del grupo estudiado fue 54,9 \pm 14 años, la edad menor fue 22 años y la mayor 74 años.

El número promedio de puntos dolorosos fue de 14,4 $\pm 1,5$. Los puntos dolorosos más frecuentes fueron los localizados a nivel del epicóndilo lateral, en el occipital y en el trapecio. Encontramos que 11 pacientes (20\%) tenían 11 de los 18 puntos dolorosos presentes, $2(3,6 \%)$ tenían 12 de los 18 puntos dolorosos presentes, 22 (40\%) 13 de los 18 puntos dolorosos, 5 (9\%) 14 de los 18 puntos dolorosos, 11 (20\%) 15 de los 18 puntos dolorosos, 11 (20\%) 16 de los 18, 2 (3,6\%) 17 de los 18 y finalmente 2 pacientes $(3,6 \%)$, los 18 puntos dolorosos presentes.
El valor promedio del puntaje de acuerdo a la Escala de Hamilton fue $10,7 \pm 6,5$; el valor mayor encontrado fue 26 y el valor menor, 2. Como mencionamos en la sección de métodos, la intensidad o severidad del ánimo depresivo depende del puntaje total de la escala de Hamilton; en los pacientes evaluados encontramos que $18(32,7 \%)$ no tenían síntomas de ánimo depresivo, en 15 $(27,7 \%)$ los síntomas fueron leves, en $17(30,9 \%)$ moderados y solo en 5 (9\%) los síntomas de ánimo depresivo fueron severos. Ningún paciente tuvo síntomas compatibles con ánimo depresivo muy severo (figura 1).

Cuando comparamos el número total de puntos dolorosos presentes en cada paciente con el puntaje encontrado en la escala de Hamilton, empleando el coeficiente de correlación de Pearson, se halló una asociación altamente positiva $(r=0,740)$ entre ambas variables. Encontramos también una asociación estadísticamente significativa $(p<0,001)$ entre el número de puntos dolorosos y los valores del puntaje total en la escala de Hamilton (figura 2).

\section{DISCUSIÓN}

La fibromialgia representa una entidad bastante frecuente, tanto en la población general como en la práctica clínica diaria; sin embargo, a pesar de su alta prevalencia, representa también una entidad poco conocida y usualmente no diagnosticada,

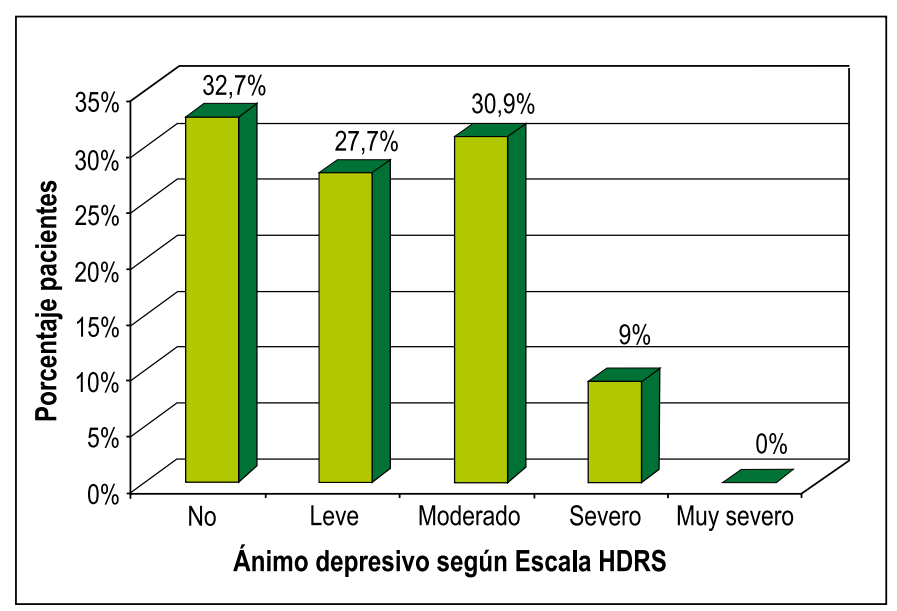

Figura 1. Ánimo depresivo de fibromialgia, evaluada mediante la Escala de Hamilton para la depresión (HDRS). 


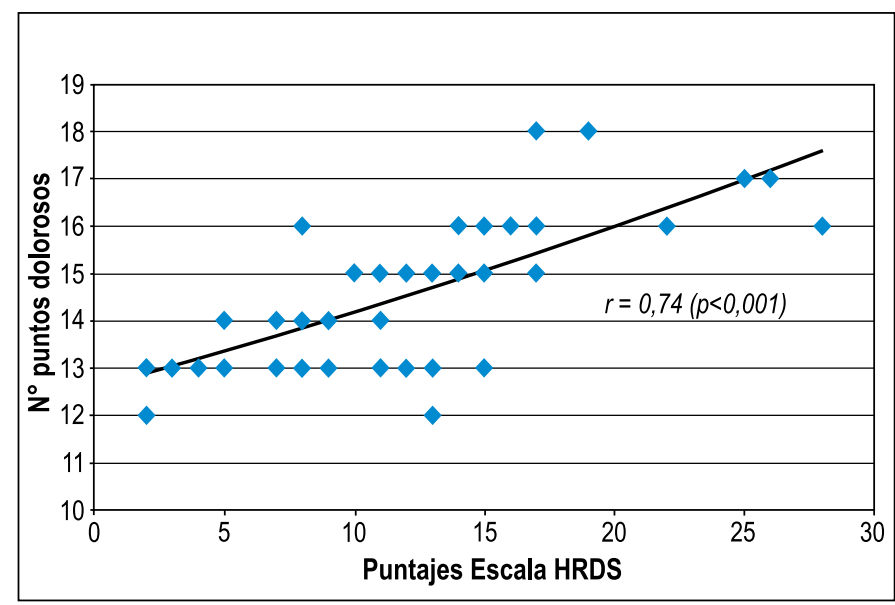

Figura 2. Correlación entre el número de 'puntos dolorosos' y el puntaje de la Escala de Hamilton para la depresión (HDRS).

incluso en países desarrollados ${ }^{(33)}$. El cuadro clínico caracterizado por síntomas en su mayoría subjetivos -con la excepción de los puntos dolorosos-, las pruebas de laboratorio dentro de límites normales y la falta de un sustrato histopatológico característico son tal vez los factores que contribuyan a que esta entidad permanezca elusiva desde el punto de vista diagnóstico ${ }^{(34)}$.

La fibromialgia es una entidad descrita como tal hace pocas décadas; sin embargo, la historia de esta entidad se remonta a varias centurias. Desde principios del siglo XVIII, los médicos empleaban el término 'reumatismo muscular' para referirse a los pacientes con dolor musculoesquelético de naturaleza no deformante y que probablemente se originaban en los tejidos blandos que rodean a las articulaciones (35). Los 'puntos dolorosos' fueron inicialmente descritos por Valleix, en $1834^{(36)}$ y, en 1904, Sir Williams Gowers empleó por primera vez el término de 'fibrositis', el cual reemplazó al término de 'reumatismo muscular' que se utilizaba hasta ese momento ${ }^{(37)}$. Posteriormente, fibrositis fue finalmente reemplazado por el término de 'fibromialgia', que es el que se emplea al momento actual para referirse a esta entidad ${ }^{(38)}$. Halliday reconoció, desde 1937, la asociación entre fibromialgia y alteraciones psicológicas, y desde ese momento una serie de publicaciones han confirmado esta asociación, en particular con trastornos depresivos, remarcando las implicaciones terapéuticas de la coexistencia de fibromialgia y trastornos depresivos ${ }^{(39)}$.
Sin embargo, en las últimas décadas se ha reconocido que los síntomas depresivos solo se presentan en una proporción de pacientes con fibromialgia. Las alteraciones psicológicas son más heterogéneas y variadas; han sido asociadas en particular con ansiedad y desórdenes de pánico ${ }^{(40-42)}$. Se reconoce actualmente que los síntomas relacionados a ansiedad se encuentran en $27 \%$ a $60 \%$ de pacientes y la probabilidad de presentar ansiedad a lo largo de la vida es $35 \%$ a $62 \%$, mientras que el desorden depresivo mayor se presenta entre $14 \%$ y $23 \%$, con una probabilidad de tener un desorden depresivo mayor a lo largo de la vida de $58 \%$ a $86 \%{ }^{(40-44)}$.

En un estudio reciente, empleando la escala HADS (hospital anxiety and depression scale), en una población importante de pacientes, Arnold encuentra una mayor prevalencia de síntomas ansiosos en relación a síntomas depresivos ( $71 \%$ versus $56 \%$ ), y el autor concluye que, en los pacientes con fibromialgia, los síntomas relacionados a ansiedad son más comunes que los síntomas depresivos ${ }^{(45)}$.

Según los resultados de nuestra investigación, encontramos que $9 \%$ de nuestros pacientes tenía al momento del diagnóstico síntomas compatibles con ánimo depresivo severo y cerca a 31\%, síntomas compatibles con ánimo depresivo moderado. Contrariamente, en más de $60 \%$ de los pacientes evaluados no encontramos síntomas de ánimo depresivo o estos síntomas de ánimo depresivo fueron leves. Estos hallazgos son compatibles con comunicaciones recientes en las cuales se señala que cerca de $30 \%$ de pacientes con fibromialgia presenta síntomas compatibles con depresión severa o moderada ${ }^{(45)}$, pero menos frecuentemente se puede encontrar un desorden depresivo mayor. Más bien, es importante considerar que fibromialgia se puede asociar con otros desórdenes psiquiátricos, en particular, con ansiedad y desórdenes de pánico, los cuales no fueron evaluados en el presente estudio ${ }^{(46)}$.

La asociación con desórdenes psiquiátricos en fibromialgia siempre han planteado la posibilidad de que estas manifestaciones sean una consecuencia del dolor crónico que experimentan los pacientes que padecen fibromialgia, más que una comorbilidad. Sin embargo, diferentes estudios han mostrado que los pacientes con fibromialgia tienen una tasa más elevada y variada de desórdenes psicológicos que los pacientes con artritis reumatoide, lo cual parece indicar que los desórdenes de la esfera mental representan más una comorbilidad que una consecuencia del dolor crónico en los pacientes con fibromialgia ${ }^{(16,47,48)}$.

En el presente estudio también encontramos que existe una correlación positiva y estadísticamente significativa entre el recuento de 'puntos dolorosos' y los síntomas de ánimo depresivo en la población de pacientes que estudiamos. Este hallazgo ya había sido previamente comunicado en la literatura; se considera que el número de 'puntos dolorosos' correlaciona con la intensidad de síntomas, en particular con ansiedad, depresión, fatiga e insomnio. Asimismo, podría reflejar el grado de extensión del problema en cada paciente en particular ${ }^{(21)}$.

Una de las limitaciones del presente estudio es que solo evaluamos síntomas de ánimo depresivo; a futuro, sería interesante evaluar la presencia de ansiedad y definir el perfil psicológico completo en nuestra población de pacientes con fibromialgia.

Concluimos que la prevalencia de síntomas depresivos severos no es frecuente en la población de pacientes con fibromialgia que hemos evaluado, pero alrededor de $30 \%$ de pacientes tuvo síntomas depresivos, usualmente leves a moderados. Estos resultados concuerdan 
con lo encontrado en la literatura, en la cual se describe que los desórdenes de la esfera mental son frecuentes y variados en fibromialgia; y también concuerda con que la depresión mayor no es la asociación más frecuente en esta entidad.

\section{REFERENCIAS BIBLIOGRÁFICAS}

1. Wolfe F, Ross K, Anderson J, Russell IJ, Hebert L. The prevalence and characteristics of fibromyalgia in the general population. Arthritis Rheum. 1995;38:19-28.

2. Croft P, Rigby AS, Boswell R, Schollum J, Silman A. The prevalence of chronic widespread pain in the general population. J Rheumatol. 1993;20:710-3.

3. Annemans L, Wessely S, Spaepen E, Caekelbergh K, Caubere JP, Le Lay K, et al. Health economic consequences related to the diagnosis of fibromyalgia syndrome. Arthritis Rheum. 2008;58:895-902.

4. Boonen A, van den Heuvel R, van Tubergen A, Goossens M, Severens JL, van der Heijde D, et al. Large differences in cost of illness and wellbeing between patients with fibromyalgia, chronic low back pain, or ankylosing spondylitis. Ann Rheum Dis. 2005;64;396-402.

5. Smythe HA, Moldofsky H. Two contributions to understanding of the 'fibrositis' syndrome. Bull Rheum Dis. 1977;28:928-31.

6. Vidal L, Hernández H, Cabello L. Síndrome de fibromialgia y reumatismo psicogénico. En: Vidal L. Bases y principios en Reumatología. 2da Ed. 1999. p. 421-44.

7. Goldenberg DL. Fibromyalgia syndrome a decade later: what have we learned? Arch Intern Med. 1999;159:777-85.

8. Mease Ph. Fibromyalgia syndrome: Review of clinical presentation, pathogenesis, outcome measures, and treatment. J Rheumatol. 2005;32 (Suppl 75):6-21.

9. Crofford LJ. The relationship of fibromyalgia to neuropathic pain syndromes. J Rheumatol. 2005;32(Suppl 75):41-5.

10. Carmonaa L, Ballinab J, Gabrielc R, Laffon A. The burden of musculoskeletal diseases in the general population of Spain: results from a national survey. Ann Rheum Dis. 2001;60:1040-5.

11. Forseth K, Gran TJ, Hisby G. A population study of the incidence of fibromyalgia among women aged 26-55 yr. Br J Rheumatol. 1997;36:1318-23.

12. Barkhuizen A. Rational and targeted pharmacologic treatment of fibromyalgia. Rheum Dis Clin North Am. 2002;28:261-90.

13. Bennett RM. The rational management of fibromyalgia patients. Rheum Dis Clin North Am. 2002;28:181-99.

14. Aguilar J, Sánchez E, Salcedo C, Burneo G, Proaño C. Estudio poblacional de frecuencia de enfermedades reumáticas en adultos mayores de 57 años. Informe Preliminar. Bol Asoc Per
Reumatol. 1994;1(3):21.

15. Vidal L, López J, Guerrero P, Farfán C, Mío F, Vargas R. Fibromyalgia syndrome: Prevalence in the Cayetano Heredia Hospital. Piura-Perú. $24^{\text {TH }}$ International Congress of Internal Medicine. IX Peruvian Congress of Internal Medicine - ISIM. Sociedad Peruana de Medicina Interna. Lima, Perú. 3-7 noviembre, 1998. p. 189.

16. Vidal Neira L, Piscoya J. Fibrositis: Estudio clínico y terapéutico. Diagnóstico. 1989;22:25.

17. Goldenberg DL. Psychologic studies in fibrositis. Am J Med. 1986;81:67-76.

18. Yunus M, Masi AT, Calabro JJ, Miller KA, Feigenbaum SL. Primary fibromyalgia (fibrositis): clinical study of 50 patients with matched normal controls. Semin Arthritis Rheum. 1981;11:151-71.

19. Smythe HA, Buskila D, Urowitz S, Langevitz P. Control and "fibrositic" tenderness; comparison of two dolorimeters. J Rheumatol. 1992;19:76872.

20.Vidal L. Síndrome de fibromialgia: diagnóstico y manejo. Rev Soc Per Med Inter. 2003;16:22-30.

23. Wolfe $F$. The relation between tender points and fibromyalgia symptom variables: evidence that fibromyalgia is not a discrete disorder in the clinic. Ann Rheum Dis. 1997;56:268-71.

21. Smythe HA, Moldofsky H. Two contributions to understanding of the fibrositis syndrome. Bull Rheum Dis. 1977;28:928-31.

22. Moldofsky H, Scarisbrick P, England R, Smythe $H$. Musculoeskeletal symptoms and NOM-REM sleep disturbance in patients with "Fibrositis syndrome" and healthy subjects. Psychosom Med. 1975;37:341-52.

24. Alpay M, Cassem EH. Diagnosis and treatment of mood disorders in patients with rheumatic disease. Ann Rheum Dis. 2000;59:2-4.

25. Arnold LM, Hudson JI, Keck PE Jr, Auchenbach MB, Hess EV. Comorbidity of fibromyalgia and psychiatric disorders. J Clin Psychiatr. 2006;67:1219-25.

26. Wolfe F, Smythe HA, Yunus MB, Bennett RM, Bombardier C, Goldenberg DL, et al. The American College of Rheumatology 1990 Criteria for the Classification of Fibromyalgia. Report of the Multicenter Criteria Committee. Arthritis Rheum. 1990;33:160-72.

27. Hamilton M. A rating scale for depression. J Neurol Neurosurg Psychiatry. 1960;23:56-62.

28. Hamilton M. Development of a rating scale for primary depressive illness. Br J Soc Clin Psychol. 1967;6:278-96.

29. Williams JB. A structured interview guide for the Hamilton Depression Rating Scale. Arch Gen Psychiatry. 1989;45:742-7.

30. Ramos-Brieva JC. A new validation of the Hamilton Rating Scale for Depression. J Psychiatr Res. 1988;22:21-8

31. Chacón V, Muñoz CX, Rivas JC, Miranda CA. Estudio descriptivo de la prevalencia de depresión en pacientes con epilepsia que consultan el Hospital Psiquiátrico Universitario del Valle (HPUV) y la Liga contra la Epilepsia (LCE) en Cali, Colombia. Rev Col Psiquiatría. 2007;36:31-40.
32. American Psychiatric Association. Handbook of psychiatric measures. Washington, DC. 2000.

33. The European Network of Fibromyalgia Associations (ENFA). Fibromyalgia Global Impact Survey Advancing Understanding, Aiding Diagnosis. Final Report. http://www.enfa-europe.eu/index1. php?id=10.

34. Vidal L, Reyes G. Fibromialgia: una entidad que debemos tener presente en la práctica clínica. Rev Cubana Reumatol. 2006;7:8-22.

35. Baldry PE. The evolution of current concepts. En: Baldry PE (ed). Myofascial Pain and Fibromyalgia Syndromes. Edinburg: Churchill Livingstone; 2001. p. 3-15.

36. Valleix FL. Traite des nervalgies ou affections douloureuses Des Nerfs. Paris: JB Bailliere; 1841. p. 266-594.

37. Gowers WR. The developement of the concept of fibrositis. Brit Med J. 1904;1:117.

38. Inanici F, Yunus MB. History of fibromyalgia: Past to present. Current Pain Headache Reports. 2004;8:369-78.

39. Halliday JL. Psychological factors in rheumatism. Brit Med J. 1937;i:213-7,264-9.

40. Goldenberg DL. Psychologic studies in fibrositis. Am J Med. 1986;81:67.

41. Walker EA, Keegan D, Gardner G, Sullivan M, Bernstein D, Katon WJ. Psychosocial factors in fibromyalgia compared with rheumatoid arthritis: I. Psychiatric diagnoses and functional disability. Psychosom Med. 1997;59:565-71.

42. Epstein SA, Kay G, Clauw D, Heaton R, Klein D, Krupp L, et al. Psychiatric disorders in patients with fibromyalgia. Psychosomatics. 1999;40:57-63.

43. Malt EA, Berle JE, Olafsson S, Lund A, Ursin H. Fibromyalgia is associated with panic disorder and functional dyspepsia with mood disorders. A study of women with random sample population controls. J Psychosom Res. 2000;49:285-9.

44. Hudson Jl, Goldenberg DL, Pope HG Jr, Keck PE $\mathrm{Jr}$, Schlesinger L. Comorbidity of fibromyalgia with medical and psychiatric disorders. Am J Med. 1992;92:363-7.

45. Arnold LM, Hudson JI, Keck PE Jr, Auchenbach MB, Hess EV. Comorbidity of fibromyalgia and psychiatric disorders. J Clin Psychiatry. 2006;67:1219-25.

46. Theme K, Turk DC, Flor H. Comorbid depression and anxiety in fibromyalgia syndrome: Relationship to somatic and psychosocial variables. Psychosomatic Med. 2004;66:837-44.

47. Alpay M, Cassem EH. Diagnosis and treatment of mood disorders in patients with rheumatic disease. Ann Rheum Dis. 2000;59:2-4.

48. Vidal L, Posadas G, Mayta M, España J, Mayta A, Cabello E. Síndrome de fibromialgia: Características clínicas. Fronteras en Medicina. 1997;5:125-31.

Manuscrito recibido el 20 de enero de 2010 y aceptado para publicación el 16 de marzo de 2010.

Correspondencia:

Dr. Luis Vidal Neira

Correo-e: Ifvidaln@hotmail.com 